\title{
Pierre Hadot 1922-2010
}

\author{
Richard Goulet \\ Centre National de la Recherche Scientifique
}

Pierre Hadot est décédé dans la nuit du 24 au 25 avril 2010 à l'âge de 88 ans. Les lecteurs de cette revue savent le rôle capital qu'il a joué dans le renouveau des études néoplatoniciennes, et connaissent très certainement ses travaux sur Plotin et Porphyre, notamment son Porphyre et Victorinus, qui retrouvait les structures de la métaphysique de Porphyre dans les traités de Marius Victorinus et proposait d'attribuer à Porphyre le commentaire anonyme sur le Parménide conservé dans le palimpseste de Turin, de même que ses pénétrantes analyses de plusieurs traités de Plotin.

De façon plus générale, de nombreux ouvrages, parfois réédités dans des collections à grande diffusion et traduits en de nombreuses langues, y compris le coréen et le finnois, ont fait de Pierre Hadot un maître internationalement reconnu qui a orienté en profondeur la conception de la philosophie et de son histoire que se sont faite ses auditeurs au cours d'une longue carrière d'enseignement, et des lecteurs plus récents aux préoccupations fort diverses. L'admiration et l'estime qu'il engendrait lui ont valu les plus insignes honneurs comme la médaille d'argent du CNRS ou le grand prix de philosophie de l'Académie française. Il fut correspondant d'académies allemandes et docteur honoris causa d'universités étrangères. Des journées d'étude consacrées à sa pensée, des articles et des monographies ont tenté de mettre en valeur l'influence qu'avaient exercée sur des penseurs contemporains ses études sur les exercices spirituels et sur la conception de l'activité philosophique dans l'antiquité. Mais cette riche et passionnante production n'était que l'aboutissement d'innombrables travaux académiques beaucoup plus austères entrepris dès après la guerre, édition critique, traduction et commentaire de quelques-uns des auteurs 
les plus riches et les plus difficiles de la philosophie antique : Marius Victorinus, Porphyre, Plotin, Épictète, Marc-Aurèle, etc. Et derrière cette production savante se cachaient encore des activités plus ingrates : collation de manuscrits, confection manuelle de lexiques, dépouillements bibliographiques, qui, sans correspondre au départ à l'approche nettement philosophique de ses premiers engagements, lui étaient apparues indispensables pour bien comprendre l'intention des textes qu'il étudiait.

Le Pierre Hadot que je voudrais évoquer à la demande de Mme Suzanne Stern-Gillet, est moins le professeur du Collège de France que le directeur d'étude de la $V^{e}$ section de l'École pratique des Hautes Études, où il enseigna de 1965 à 1985. C'est la lecture de Plotin ou la simplicité du regard qui m'amena à Paris en 1969. Les conférences de l'EPHE à cette époque ne présentaient pas le caractère protocolaire, pour ne pas dire mondain, des cours du Collège de France. Quinze ou vingt auditeurs de tous les âges, venus d'un peu partout, y compris quelques originaux à qui il valait mieux ne pas laisser la parole trop longtemps, prenaient place, le lundi soir, dans la grande salle mal éclairée de la Section où l'on prenait des notes sur des tables qui avaient la forme d'étroites planches de bois. Le conférencier essayait de couvrir le bruit de la circulation de la rue Saint-Jacques ou d'écrire sur un tableau vert où les mots se distinguaient à peine. D'autres conférences étaient données autour d'une table dans le "laboratoire" enfumé, car c'était une époque où on laissait encore les auditeurs fumer en salle de cours. La direction d'étude à laquelle Pierre Hadot avait été élu portait au début comme intitulé Patristique latine et on y commentait notamment les Confessions de saint Augustin. Par la suite, elle fut réintitulée Théologies et mystiques de la Grèce hellénistique et de la fin de l'antiquité et de nouveaux thèmes furent développés et de nouveaux textes furent abordés, d'abord les traités de Plotin, puis les Pensées de MarcAurèle, les aspects concrets de la vie des écoles philosophiques, la conception de la philosophie dans le testament et les lettres d'Épicure, les parties de la philosophie, la division aristotélicienne des sciences, la rhétorique antique, la logique stoïcienne, les voies et les lois de la nature, chantiers qui allaient fournir la matière des nombreuses publications de la période ultérieure. Chaque année deux thèmes de conférences distincts étaient abordés. Sur tous ces chantiers qui se renouvelaient annuellement, Pierre Hadot apportait une ample information et une grande rigueur dans l'analyse des textes. Non seulement il en maîtrisait parfaitement la 
bibliographie, mais il nous étonnait constamment par d'innombrables références aux poètes ou philosophes allemands, à des philosophes français dont nous ne connaissions parfois que les noms, à certains maîtres de la sagesse orientale et même à des peintures dont il savait exploiter la signification philosophique, comme par exemple l'analyse qu'il donnait de la Bataille d'Alexandre d'Altdorfer pour illustrer le thème du regard d'en haut.

Il n'est pas sûr que les auditeurs de l'EPHE aient perçu à l'époque combien ces divers sujets de recherche étaient choisis en fonction de préoccupations philosophiques très personnelles qui avaient inspiré les réflexions de Pierre Hadot depuis le début de sa carrière: la signification de l'expérience mystique, le rôle de la philosophie et la tâche du philosophe, les principes de la morale et, bien sûr, l'importance des exercices spirituels, qui était chez les Hadot un thème en quelque sorte conjugal, puisqu'il avait fait également l'objet des travaux de Madame Hadot.

Conformément aux principes de l'EPHE, les conférences de P. Hadot étaient le résultat des recherches en cours du directeur d'étude. Ceux qui attendaient des dissertations bien structurées n'y trouvaient pas nécessairement leur compte. De semaine en semaine, P. Hadot ajoutait des pages sur les petits classeurs à anneaux qu'il utilisait et en restituait le contenu à ses auditeurs. Prendre en notes ces cours hebdomadaires qui se déroulaient au plus près des textes n'était pas facile et il était préférable d'en réécrire le contenu au retour à la maison, en intégrant le texte des nombreuses citations qui avaient été prises en compte.

Les directeurs de l'École pratique avaient en général le souci de prendre à la lettre le qualificatif de "pratique" qui faisait en principe l'originalité de cette institution, laquelle, encore à cette époque, se bornait à décerner des "diplômes" qui pouvaient faire 50 pages aussi bien que 900. Les conférences n'étaient pas des cours magistraux et les auditeurs pouvaient poser des questions et intervenir, parfois même préparer un exposé sur un sujet connexe. J'avais été moi-même honoré et surtout intimidé d'avoir à traiter de la théorie stoïcienne des propositions dans le cadre du séminaire et encore plus d'avoir à développer cet exposé au Colloque de Chantilly sur la logique stoïcienne où $\mathrm{M}$. Hadot mavait fait inviter avec d'autres membres du séminaire. Certaines années, une charge de conférence complémentaire était confiée à des élèves de la direction d'études. Après avoir suivi les deux heures régulières de conférence, il fallait soi-même traiter son sujet devant M. et Mme Hadot, ainsi que devant le cercle habituel 
d'auditeurs qui comprenait des chercheurs confirmés. La première année, j'avais déversé dès la première heure de conférence toute la matière que j'avais préparée au cours de l'été et il me fallut par la suite, de semaine en semaine, trouver la substance du cours du lundi suivant.

Les cours de P. Hadot étaient également des leçons de méthode et implicitement des prises de position contre certaines déviations de la pratique de l'histoire de la philosophie. A plusieurs reprises, il avait obtenu que les conférences soient complétées par des cours de méthodologie. C'est ainsi qu'il avait demandé au Père Michel Aubineau en 1969-1970 de nous initier à divers outils de documentation, notamment aux corpus d'inscriptions, aux instruments bibliographiques, mais aussi à la lecture des manuscrits grecs. Une autre année, il commença lui-même à assurer un séminaire de formation à l'allemand philosophique, expérience qui fut interrompue par les interventions d'un jeune étranger germanophone qui compromettait l'esprit de l'entreprise en essayant de montrer de façon intempestive qu'il maîtrisait lui-même parfaitement la langue.

Préparer une thèse ou un mémoire à la $V^{e}$ section, c'était à l'époque rencontrer son directeur de thèse deux ou trois fois par année debout dans un couloir ou bien dans le petit bureau de trois ou quatre mètres carrés qui était partagé par tous les directeurs. Mais pour des discussions plus importantes Pierre Hadot nous invitait chez lui, à Parmain, puis plus tard à Limours. Il lisait les pages qu'on lui apportait, repérait immédiatement les points forts et les faiblesses de l'argumentation et exprimait un point de vue susceptible de réorienter le travail pour plusieurs mois.

Pour un étranger ou un étudiant français qui n’avait pas suivi la filière classique de l'École normale et de l'agrégation, entrer au CNRS était une entreprise très difficile. Peut-être parce que lui-même avait suivi une formation et une carrière fort mouvementées (qu'il a racontées dans son livre d'entretiens avec Jeannie Carlier et Arnold Davidson), P. Hadot ne ménageait pas sa peine pour soutenir ses candidats et nous sommes quelques collègues à devoir notre poste de chercheur à la confiance qu'il nous a accordée. Il avait lui-même commencé sa carrière au CNRS et y était resté seize ans, à une époque où le maintien dans le cadre des chercheurs était remis en question chaque année.

Lorsqu'en 1981 je lui présentai le projet d'un répertoire prosopographique de tous les philosophes grecs et romains qui prendrait en compte les sources épigraphiques ou papyrologiques et qui recenserait leurs 
œuvres conservées ou seulement attestées, il en approuva immédiatement l'idée et accepta de rédiger une préface substantielle qui en décrivit les objectifs et les grandes perspectives. Par la suite, il contribua à ce Dictionnaire des philosophes antiques par une liste savamment commentée des titres d'ouvrages de Chrysippe.

Sa bonté, sa simplicité, sa sérénité et aussi son humour, lui ont valu l'amitié de nombreux universitaires et chercheurs du monde entier, célèbres ou encore inconnus. La maison de Limours et son splendide jardin ont accueilli d'innombrables collègues et étudiants et ont été le lieu de fructueux échanges.

Pour ses proches et ses amis, la santé de Pierre Hadot fut un sujet de préoccupation constante. Pour nous rassurer, lors de sa première opération à cœur ouvert, où on lui avait greffé une valve artificielle, il nous disait que les médecins pensaient que l'appareillage pourrait très bien tenir au moins dix ans. Heureusement, ce délai fut largement dépassé, mais ce fut au prix de plusieurs nouvelles opérations lourdes qui l'ont sauvé de situations périlleuses, sans pouvoir empêcher une anémie persistante qui aurait pu faire baisser les bras à un homme moins courageux. Mais, grâce à l'assistance indéfectible de son épouse, il surmonta toutes ces épreuves et il maintint un rythme de travail impressionnant jusqu'à sa disparition, malgré une vue déclinante, s'efforçant même d'apprendre à manier les outils informatiques indispensables. Il n'hésitait pas, encore ces dernières années, à entreprendre des voyages pour travailler dans des bibliothèques étrangères ou simplement pour faire visiter à son petit-fils Adrien quelque site qu'il affectionnait.

Lorsque l'on voit l'impressionnante production scientifique de Pierre Hadot et la renommée internationale qu'elle lui a value, on ne peut pas ne pas constater qu'une telle activité de recherche, unifiée, cohérente, planifiée, s'est déployée dans un cadre essentiellement individuel, à l'écart des colloques, sans concession aux recueils de mélanges in honorem (lui-même a toujours refusé qu'on lui offrît un volume d'hommages), et sans recourir ni participer aux programmes de recherche, nationaux ou internationaux, bien délimités dans le temps et financés à coup de milliers d'euros, que l'on voudrait aujourd'hui imposer comme cadre indispensable de la recherche, y compris en sciences humaines. C'est là un exemple sur lequel les décideurs qui entendent piloter et évaluer la recherche devraient aujourd'hui réfléchir. 